\title{
Naringin Suppresses Osteoclast Formation and Enhances Bone Mass in Mice
}

\author{
Michiko Hirata, Chiho Matsumoto, Morichika Takita, Chisato Miyaura, ${ }^{*}$ and Masaki Inada \\ Department of Biotechnology and Life Science, Tokyo University of Agriculture and Technology, 2-24-16 Nakamachi, Koganei, Tokyo \\ 184-8588, Japan
}

(Received January 12, 2009; Accepted January 27, 2009)

\begin{abstract}
Naringin is a flavonoid commonly found in citrus fruits. Previous studies have supported a positive association between fruit consumption and health including bone quality. To clarify the role of naringin in bone turnover, we examined the effect of naringin on bone mass in mice, and on osteoclast formation induced by bone-resorbing cytokine. When mice were orally administered naringin for 2 weeks, femoral bone mineral density (BMD) was clearly elevated compared with control mice. BMD in distal and middle portion was significantly enhanced, but proximal BMD was not changed by naringin administration. In soft-X-ray analysis, a marked accumulation of cancellous bone could be detected in distal femoral metasphysis in mice treated with naringin. In cocultures of bone marrow cells and osteoblasts, naringin dose-dependently suppressed the number of osteoclasts formed by treatment with interleukin-1 (IL-1). The size of osteoclasts formed in the presence of naringin was smaller than that induced by IL-1. Naringin enhances bone mass possibly by suppression of osteoclast formation in mice, suggesting the role of the citrus flavonoid on bone health and to prevent bone diseases such as osteoporosis.
\end{abstract}

Key words — bone mass, osteoclast, naringin, citrus flavonoid

\section{INTRODUCTION}

Bone remodeling is regulated by osteoclastic bone resorption and new bone formation. Osteocalsts are primary bone-resorbing cells, and are differentiated from the monocyte-macrophage lineage.

\footnotetext{
*To whom correspondence should be addressed: Department of Biotechnology and Life Science, Tokyo University of Agriculture and Technology, 2-24-16 Nakamachi, Koganei, Tokyo 184-8588, Japan. Tel. \& Fax: +81-42-388-7390; E-mail: miyaura@cc.tuat.ac.jp
}

As a pivotal factor in osteoclast differentiation, previous studies have identified the receptor activator of Nuclear Factor (NF)- $\kappa$ B ligand (RANKL), also known as the osteoprotegerin (OPG) ligand. ${ }^{1-3)}$ Osteoblasts express RANKL in response to bone-resorbing factors such as interleukin-1 (IL-1), parathyroid hormone (PTH), and $1 \alpha, 25-$ dihydroxyvitamin $\mathrm{D}_{3}\left[1 \alpha, 25(\mathrm{OH})_{2} \mathrm{D}_{3}\right]$, and interact with osteoclast precursors expressing RANK, inducing differentiation into mature osteoclasts via a mechanism of RANK-RANKL recognition. In new bone formation, the proliferation and differentiation of osteoblastic cells are critical to enhance bone formation. Bone morphogenetic proteins (BMPs) and insulin-like growth factors (IGFs) are important regulators in the proliferation and differentiation of osteoblastic cells. ${ }^{4)}$ Bone remodeling is regulated by several systemic hormones such as PTH, $1 \alpha, 25(\mathrm{OH})_{2} \mathrm{D}_{3}$, calcitonin, and sex hormones. On the other hand, local factors such as IGFs, BMPs, prostaglandins, and cytokines are also important for the maintenance of bone tissues. The proportion of bone resorption and formation is essential to keep good bone quality and high bone mass.

Naringin is a flavonoid commonly found in citrus fruits such as grapefruit peel. ${ }^{5)}$ It has been reported that naringin exhibits anti-oxidant and anticholesterol effects. In cholesterol metabolism, naringin is known to act as an inhibitor for a hydroxymethylglutaryl coenzyme A (HMG-CoA) reductase, the rate-limiting enzyme in the mevalonate pathway. ${ }^{6}$ In addition, epidemiological studies have supported a positive association between fruit consumption and health including bone quality. ${ }^{7)}$ Recent studies have shown that naringin enhanced the proliferation of various osteoblastic cells including rat osteosarcoma cells (UMR106), mouse osteoblastic cells (MC3T3-E1), and primary mouse osteoblastic cells. ${ }^{8,9)}$ Mundy et al. ${ }^{10)}$ have reported that statin, a HMG-CoA reductase in- 
hibitor, enhances BMP-2 expression and stimulates bone formation in bone tissues. Wu et al. ${ }^{8)}$ reported that naringin enhanced BMP-2 expression in osteoblasts and stimulated the cell growth of osteoblasts. Therefore it is possible that naringin acts on osteoblasts to enhance bone formation by inhibiting HMG-CoA reductase in osteoblasts.

On the other hand, a possible role of naringin in bone resorption has not been reported. Recently, Mandadi et al. ${ }^{11)}$ have reported that administration of naringin suppressed the plasma total resistant acid phosphatase, a marker of bone resorption, in osteoporosis rats induced by orchidectomy. Therefore it is possible that naringin modulates bone resorption in bone tissue. In the present study, we examined the influence of naringin in osteoclast differentiation in vitro and in bone mass in vivo to define a possible role of the citrus-derived flavonoid in bone tissues.

\section{MATERIALS AND METHODS}

Animals and Reagents — Newborn, 4-weekold, and 6-week-old mice $d d y$ strains were obtained from Japan SLC Inc. (Shizuoka, Japan). Four-weekold mice were fed a laboratory chow containing $1.12 \%$ calcium and $1.07 \%$ phosphorus (Nippon Clea, Tokyo, Japan) for 2 weeks. For the treatment of naringin, the mice were fed with a chow containing $3.5 \%$ of naringin. The mean intake of naringin was $140 \mathrm{mg} / \mathrm{mouse}$ per day. The mice were killed to collect the femurs. Naringin, purified form citrus fruit (chemical purity $>98 \%$ ), was purchased from LKT Laboratories Co. Ltd. (St. Paul, MN, U.S.A.). Recombinant human IL- $1 \alpha$ was purchased from R\&D Systems (Minneapolis, MN, U.S.A.). Animal care and experiments were carried out in accordance with our institutional animal care regulations.

Radiographic Analysis of the Femur — Radiographs of the femurs were taken with soft-X-ray (model CMB-2; SOFTEX, Tokyo, Japan). The bone mineral density (BMD) of the femurs was measured by dual X-ray absorptiometry (model DCS-600R; Aloka, Tokyo, Japan), as reported previously. ${ }^{12)}$ The bone mineral content (BMC) of the mouse femur was closely correlated with the ash weight. The BMD was calculated by dividing the BMC of the measured area by the total area. The scanned area was dividing equally into three regions comprising the proximal, middle, and distal femur to assess re- gional differences.

Osteoclast Formation in Coculture of Mouse Bone Marrow Cells and Osteoblasts - Primary osteoblastic cells were isolated from newborn mouse calvariae after five routine sequential digestions with $0.1 \%$ collagenase (Wako, Osaka, Japan) and $0.2 \%$ dispase (Godo Shusei, Tokyo, Japan). Osteoblastic cells collected from fractions 2-4 were combined and cultured in $\alpha$-modified MEM ( $\alpha$ MEM), supplemented with $10 \%$ fetal calf serum (FCS) at $37^{\circ} \mathrm{C}$ under $5 \% \mathrm{CO}_{2}$ in air. Bone marrow cells $\left(3 \times 10^{6}\right.$ cells $)$ were isolated from 6 week-old mice and cocultured with the primary osteoblastic cells $\left(1 \times 10^{4}\right.$ cells $)$ in $\alpha$ MEM containing $10 \%$ FCS. After culturing for 7 days, the cells adhering to the well surface were stained for tartrateresistant acid phosphatase (TRAP). TRAP-positive multinucleated cells containing three or more nuclei per cell were counted as osteoclasts.

Statistical Analysis — Data are expressed as the means \pm standard error of the mean (S.E.M.). The significance of differences was analyzed by Student's $t$ test.

\section{RESULTS}

Naringin is a flavonoid commonly found in citrus fruits (Fig. 1). Naringin used in the present study was purified from citrus fruit, and the purity was $>98 \%$.

To define the effects of naringin on bone mass in vivo, we first examined the effects of naringin on bone mass in mice. Four-week-old female mice were fed a control chow or naringin-containing chow for 2 weeks. The mean intake of the chow was $4 \mathrm{~g} /$ day in both control and naringin-treated mouse. Therefore the mean amount of naringin treatment was $140 \mathrm{mg} /$ mouse per day. Figure $2 \mathrm{~A}$ shows the body weight of mice at the end of the experiments.

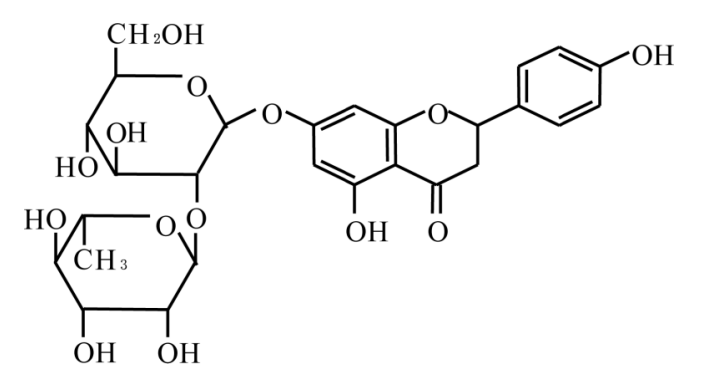

Fig. 1. Structure of Naringin 
A

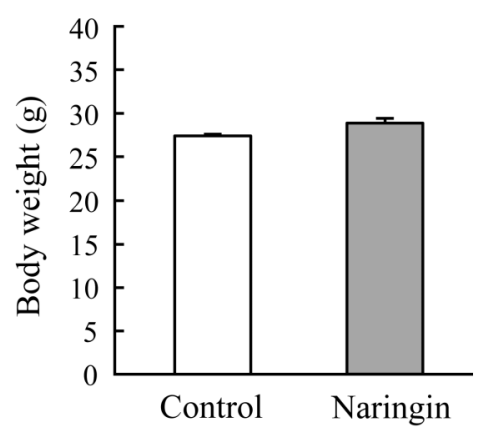

B

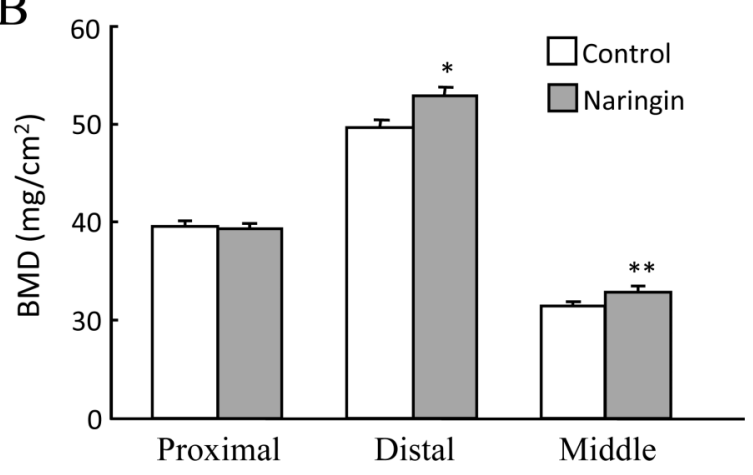

Fig. 2. Influence of Naringin Treatment on Body Weight and Femoral BMD

Four-week-old female mice were fed a control chow or naringincontaining chow for 2 weeks. The mean intake of naringin was $140 \mathrm{mg} / \mathrm{mouse}$ per day. After the feeding, body weight (A) and femoral BMD (B) were measured. In the measurement of BMD, the scanned area was divided equally into three regions comprising the proximal, middle, and distal femur. ${ }^{*} p<0.05 ;{ }^{* *} p<0.005$ versus control. Data are expressed as means \pm S.E.M. of 8 mice.

Body weight was not influenced by the treatment of naringin. Then, mice were killed to collect the femurs for radiographic analyses. When we measured BMD of the femurs, the scanned area was dividing equally into three regions comprising the proximal, middle, and distal femur to assess regional differences. The treatment of naringin significantly stimulated the distal and middle BMD, but the proximal BMD was not influenced by the treatment of naringin (Fig. 2B). There is a large amount of cancellous bone in the portion of distal femur. In contrast, the middle portion of femur consists of cortical bone without cancellous bone. In soft-X-ray analysis, the length was same in the femurs of 2 groups. The naringin-treated mice showed increased bone mass in femur, and the mass of cancellous bone in distal femur was markedly enhanced compared with control (Fig. 3). These results indicate that naringin influences both cancellous bone and cortical bone to elevate bone mass in mice.

Next, we examined the effects of naringin on osteoclast differentiation in cocultures of mouse bone
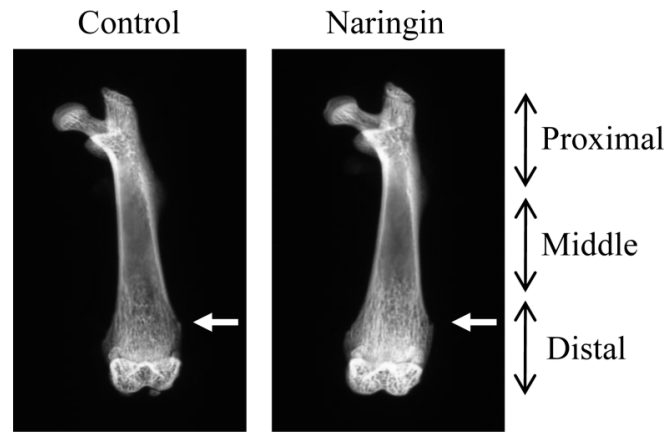

Fig. 3. Soft-X-ray Analysis of Femoral Bone Mass

Four-week-old female mice were fed a control chow or naringincontaining chow for 2 weeks. After the feeding, femurs were collected and soft-X-ray analysis was performed. Arrows indicate the portion of distal femur with rich cancellous bone.

marrow cells and osteoblasts. In the cocultures, IL-1 markedly induced osteoclast formation, and naringin dose-dependently and significantly suppressed the osteoclast formation induced by IL-1 (Fig. 4). In addition to the changes in osteoclast number, the size was also changed by the treatment of naringin. The size of osteoclasts formed in the presence of naringin was smaller than that induced by IL-1, as shown in the upper panel of Fig. 4. Naringin did not influence osteoclast formation in the absence of IL-1.

\section{DISCUSSION}

Recent studies have shown that naringin acts on osteoblasts and stimulates cell growth and differentiation. In osteoblasts, naringin stimulates the expression of BMP-2, osteocalcin, and alkaline phosphatase. ${ }^{8,9)}$ It is known that naringin acts as an inhibitor for HMG-CoA reductase, and that statin, a HMG-CoA reductase inhibitor, enhances BMP-2 expression and stimulates bone formation in bone tissues. ${ }^{6,10)}$ Therefore it is possible that naringin stimulates bone formation by a mechanism including the mevalonate pathway. In the present study, we showed that naringin suppresses osteoclast differentiation and stimulates bone mass in normal mice in vivo. Recently $\mathrm{Wu}$ et al. ${ }^{8)}$ reported that naringin partially enhanced bone mass in ovariectomized (OVX) mice, which is a typical model of postmenopausal osteoporosis due to estrogen deficiency. In their paper, they did not perform the treatment of naringin in sham-operated mouse. In the present study, we showed that naringin significantly enhances femoral bone mass in normal 

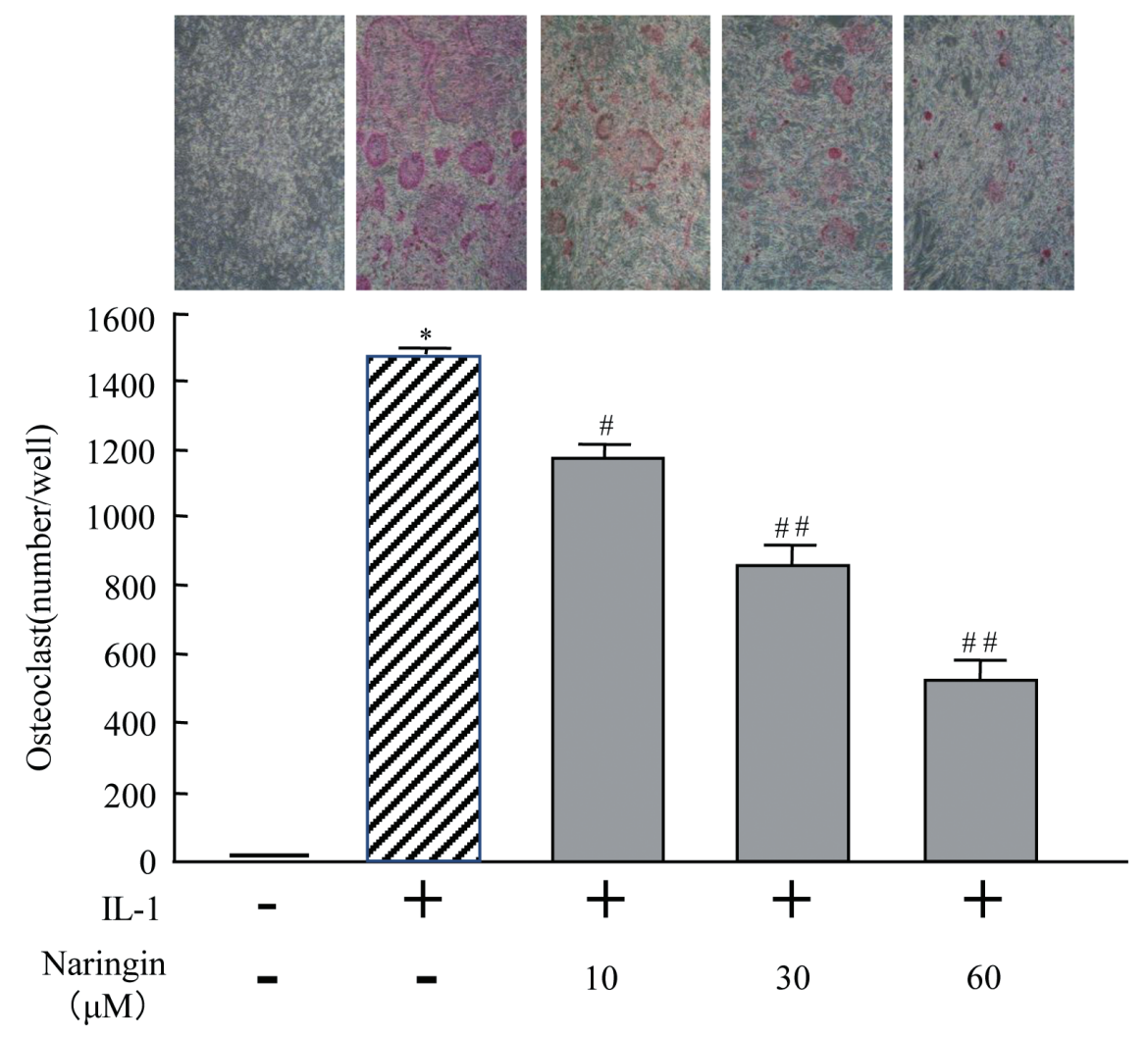

Fig. 4. Effect of Naringin on Osteoclast Formation Induced by IL-1 in Cocultures of Mouse Bone Marrow Cells and Osteoblasts

Mouse bone marrow cells were cocultured with primary mouse osteoblasts in the presence or absence of IL-1 ( $2 \mathrm{ng} / \mathrm{ml})$ with or without naringin $(10-60 \mu \mathrm{M})$ for 7 days. The number of TRAP-positive osteocalsts was counted. The upper panels indicate the representative field of a respective culture. Data are expressed as means \pm S.E.M. of 3 wells. ${ }^{*} p<0.001$ versus control; $\# p<0.01$ versus IL-1; \#\#p 0.001 versus IL-1.

mouse. Therefore it is possible that naringin enhances bone mass in both sham-operated and OVX mouse. Bone loss in OVX mouse is due to elevation of osteoclastic bone resorption, and naringin markedly suppressed osteoclast formation shown in the present study. Therefore it is possible that naringin enhances bone mass by suppressing bone resorption.

Osteocalsts are differentiated from the monocytemacrophage lineage. As a pivotal factor in osteoclast differentiation, previous studies have identified RANKL, which is expressed on the cell surface of osteoblasts in response to bone-resorbing factors. ${ }^{1-3)}$ Osteoclast precursors possess RANK, and can differentiate into mature osteoclasts via a mechanism of RANK-RANKL recognition. Most bone-resorbing factors such as PTH, IL-1, $1 \alpha$, $25(\mathrm{OH})_{2} \mathrm{D}_{3}$, and prostaglandin $\mathrm{E}$ act on osteoblasts to express RANKL to elicit osteoclast differentiation. In addition, recent studies have shown that RANKL binding to RANK results in the recruitment of tumor necrosis factor receptor-associated factor 6 (TRAF6) in osteoclast precursors, and the TRAF6-dependent signal elicits the induction of a nuclear factor of activated $\mathrm{T}$ cells cytoplasmic 1 (NFATc1), which acts as a master transcription factor for osteoclastogenesis. ${ }^{13)}$ In the present study, we showed that naringin suppressed osteoclastogenesis induced by IL-1. It is not known whether naringin regulates RANKL expression in osteoblasts, or modulates TRAF6-dependent expression of NFATc1 in osteoclast precursors. The mechanism of action of naringin in osteoclastogenesis is under investigation in our laboratory.

Epidemiological studies support a positive association between increased consumption of fruits and health, including bone quality. ${ }^{7)}$ A previous study showed that citrus significantly improves bone density of spine and femur in rats, suggesting that factors including citrus exhibit bone protective property. ${ }^{14)}$ Chiba et al. ${ }^{15)}$ reported that administration of hesperidin, a citrus flavonoid, significantly prevented bone loss in OVX mice. In addition, citrusderived carotenoid, $\beta$-cryptoxanthin, is known to suppress bone resorption and prevent bone loss in osteoporosis in animals and human. ${ }^{16)}$ Therefore several biological factors may be involved in the effects of citrus in bone tissues. Further studies 
are needed to define the role of naringin in bone metabolism and in prevention of bone diseases such as osteoporosis.

\section{REFERENCES}

1) Wong, B. R., Rho, J., Arron, J., Robinson, E., Orlinick, J., Chao, M., Kalachikov, S., Cayani, E., Bartlett, F. S., III, Frankel, W. N., Lee, S. Y. and Choi, Y. (1997) TRANCE is a novel ligand of the tumor necrosis factor receptor family that activates c-Jun N-terminal kinase in T cells. J. Biol. Chem., 272, 25190-25194.

2) Lacey, D. L., Timms, E., Tan, H. L., Kelley, M. J., Dunstan, C. R., Burgess, T., Elliott, R., Colombero, A., Elliott, G., Scully, S., Hsu, H., Sullivan, J., Hawkins, N., Davy, E., Capparelli, C., Eli, A., Qian, Y. X., Kaufman, S., Sarosi, I., Shalhoub, V., Senaldi, G., Guo, J., Delaney, J. and Boyle, W. J. (1998) Osteoprotegerin ligand is a cytokine that regulates osteoclast differentiation and activation. Cell, 93, 165-176.

3) Yasuda, H., Shima, N., Nakagawa, N., Yamaguchi, K., Kinosaki, M., Mochizuki, S., Tomoyasu, A., Yano, K., Goto, M., Murakami, A., Tsuda, E., Morinaga, T., Higashio, K., Udagawa, N., Takahashi, N. and Suda, T. (1998) Osteoclast differentiation factor is a ligand for osteoprotegerin/ osteoclastogenesis-inhibitory factor and is identical to TRANCE/RANKL. Proc. Natl. Acad. Sci. U.S.A., 95, 3597-3602.

4) Chen, D., Zhao, M. and Mundy, G. R. (2004) Bone morphogenetic proteins. Growth Factors, 22, 233241.

5) Swiader, K. E. and Zarawska, E. (1996) Flavonoids of rare Artemesia species and their antifungal properties. Fitoterapia, 67, 77-78.

6) Sifton, D. W. (2000) Zocor. N. Physicians' Desk Reference (Sifton, D. W., Ed.), Medical Economics Company, Montvale, NJ, U.S.A., pp. 1917-1920.

7) Chen, Y. M., Ho, S. C. and Woo, J. L. (2006) Greater fruit and vegetable intake is associated with increased bone mass among postmenopausal Chinese women. Br. J. Nutr., 96, 745-751.

8) Wu, J. B., Fong, Y. C., Tsai, H. Y., Chen, Y. F., Tsuzuki, M. and Tang, C. H. (2008) Naringininduced bone morphogenetic protein-2 expression via PI3 K, Akt, c-Fos/c-Jun and AP-1 pathway in osteoblasts. Eur. J. Pharmacol., 588, 333-341.

9) Wong, R. W. and Rabie, A. B. (2006) Effect of naringin on bone cells. J. Orthop. Res., 24, 20452050.

10) Mundy, G., Garrett, R. and Harris, S. (1999) Stimulation of bone formation in vitro and in rodents by statins. Science, 286, 1946-1949.

11) Mandadi, K., Ramirez, M., Jayaprakasha, G. K., Faraji, B., Lihono, M., Deyhim, F. and Patil, B. S. (2008) Citrus bioactive compounds improve bone quality and plasma antioxidant activity in orchidectomized rats. Phytomedicine, in press [Epub ahead of print].

12) Miyaura, C., Inada, M., Matsumoto, C., Ohshiba, T., Uozumi, N., Shimizu, T. and Ito, A. (2003) An essential role of cytosolic phospholipase $\mathrm{A} 2 \alpha$ in prostaglandin E2-mediated bone resorption associated with Inflammation. J. Exp. Med., 197, 13031310.

13) Nakashima, T. and Takayanagi, H. (2008) The dynamic interplay between osteoclasts and the immune system. Arch. Biochem. Biophys., 473, 166-171.

14) Deyhim, F., Garica, K., Lopez, E., Gonzalez, J., Ino, S., Garcia, M. and Patil, B. S. (2006) Citrus juice modulates bone strength in male senescent rat model of osteoporosis. Nutrition, 22, 559-563.

15) Chiba, H., Uehara, M., Wu, J., Wang, X., Masuyama, R., Suzuki, K., Kanazawa, K. and Ishimi, Y. (2003) Hesperidin, a citrus flavonoid, inhibits bone loss and decreases serum and hepatic lipids in ovariectomized mice. J. Nutr., 133, 18921897.

16) Sugiura, M., Nakamura, M., Ogawa, K., Ikoma, Y., Ando, F. and Yano, M. (2008) Bone mineral density in post-menopausal female subjects is associated with serum antioxidant carotenoids. Osteoporos. Int., 19, 211-219. 